\title{
Longikaurin A, a natural ent-kaurane, suppresses stemness in nasopharyngeal carcinoma cells
}

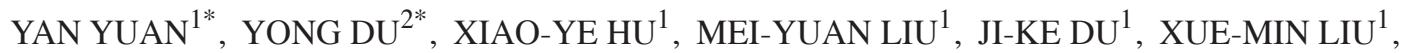 \\ HONG-EN YU ${ }^{1}$, TIAN-ZHU WANG ${ }^{1}$, JIAN-XIN PU ${ }^{3}$, QIAN ZHONG ${ }^{2}$ and QING-FENG ZOU ${ }^{1}$ \\ ${ }^{1}$ Section 3 of Internal Medicine, Cancer Center of Guangzhou Medical University, Guangzhou, Guangdong 510095; \\ ${ }^{2}$ State Key Laboratory of Oncology in South China, Collaborative Innovation Center for Cancer Medicine, \\ Sun Yat-sen University Cancer Center, Guangzhou, Guangdong 510060; ${ }^{3}$ Kunming Institute of Botany, \\ Chinese Academy of Science, Kunming, Yunnan 650000, P.R. China
}

Received May 13,2015; Accepted September 27, 2016

DOI: $10.3892 / 01.2017 .5625$

\begin{abstract}
Cancer stem cells (CSCs) are a small proportion of tumor cells that may be responsible for tumor metastasis and recurrence. Our recent research indicated that longikaurin A (LK-A) exhibited anti-tumor activity in nasopharyngeal carcinoma (NPC) both in vitro and in vivo. Here, we further investigated whether LK-A could suppress the stemness of NPC cells. Sphere formation assay was used to assess the self-renewal ability of the cells treated with LK-A. Side population (SP) was determined by flow cytometry to measure the influence of LK-A on NPC SPs. The expression of the c-myc and fibronectin was detected by western blotting. The cytotoxicity of LK-A in combination with cisplatin to NPC cells was determined by MTT assay. Colony formation assay was used to verify whether LK-A could sensitize NPC cells to radiation and reverse the radiotherapy resistance. In the present study, we found that LK-A reduced the number and size of spheroid formation and decreased the SP cell percentage of the S18 cell line at a low concentration. Furthermore, LK-A treatment downregulated the expression of c-myc and fibronectin in NPC cell lines. Moreover, LK-A could significantly enhance the chemotherapeutic and radiotherapeutic sensitivity of NPC cell lines and reverse acquired radiotherapy resistance
\end{abstract}

Correspondence to: Professor Qing-Feng Zou, Section 3 of Internal Medicine, Cancer Center of Guangzhou Medical University, 78 Hengzhigang, Guangzhou, Guangdong 510095, P.R. China E-mail: qingfeng9829@163.com

Dr Qian Zhong, State Key Laboratory of Oncology in South China, Collaborative Innovation Center for Cancer Medicine, Sun Yat-sen University Cancer Center, 651 Dongfeng Road East, Guangzhou, Guangdong 510060, P.R. China

E-mail: zhongqian@sysucc.org.cn

*Contributed equally

Key words: longikaurin A, nasopharyngeal carcinoma, cancer stem cells of Sune2-IR. Our data revealed that LK-A could suppress the stemness of NPC cells and may enhance the efficacy of radiotherapy and chemotherapy.

\section{Introduction}

Nasopharyngeal carcinoma (NPC) is one of the most common malignant tumors in South China (1). In 2010, an estimated 41,503 new cases and 20,058 deaths were attributed to NPC in China, accounting for $1.03 \%$ of all cancer-related deaths that year in China (2). Among the head and neck tumors, NPC has the highest propensity to metastasize to distant sites (3). Although the standard treatment has improved the local control rate for NPC from 54 to $78 \%$, regional recurrences occur in $10-19 \%$ of patients (4). Most of the regional recurrences are due to the metastasis and therapy resistance (1). It has been proved that cancer stem cells (CSCs) play a major role in tumor recurrence, metastasis and therapy resistance (5).

The CSC hypothesis proposes that a small subset of cancer cells has the properties of self-renewal, differentiation and resistance to chemotherapy or radiotherapy (6). Although the CSCs possess the capacity to form the heterogeneous lineages of cancer cells that comprise the tumor (6), the methods to divide the CSCs from normal cancer cells have not been developed. However, previous studies showed that CSCs expressed one or several surface markers, including cluster of differentiation (CD) 24 (7), CD44 (8), aldehyde dehydrogenase 1 (9) and CD133 (10), in particular human cancers. However, there is not a reliable CSC marker in NPC cells, and the side population (SP) phenotype can be a marker of CSCs (11) and gastrointestinal cancer (12). Therefore, effective compounds that target NPC CSCs may be helpful in clinical therapy.

Several studies have revealed that nigericin (13), and Smac mimetics in combination with TNF-related apoptosis-inducing ligand (14) could target NPC CSCs. Longikaurin A (LK-A) is a natural ent-kaurene diterpenoid extracted from the Isodon genus whose anti-tumor activity has been verified $(15,16)$. According to our previous research, at a low concentration, LK-A could inhibit the colony formation ability of NPC cells (15). In addition, LK-A exhibits anti-tumor activity in CNE2 xenograft tumor models (15). However, to the best of 
our knowledge, no research has been conducted to examine the effect of LK-A on CSCs. Here, we studied whether LK-A could suppress stemness in NPC.

\section{Materials and methods}

Chemicals and antibodies. We used a previously described method to obtain LK-A from the leaves of Isodon ternifolius (D. Don) Kudô (15). Briefly, the dried and milled plant material $(10 \mathrm{~kg})$ was extracted four times by incubation with 1001 of $70 \%$ aqueous $\mathrm{Me}_{2} \mathrm{CO}$ for 3 days at room temperature and then filtered. The filtrate was evaporated under reduced pressure, and partitioned with ethyl acetate (EtOAc) (4x60 1). The EtOAc partition (938.5 g) was applied to a silica gel (200-300 mesh), and six fractions, termed A-F, were eluted with $\mathrm{CHCl}_{3}-\mathrm{Me}_{2} \mathrm{CO}$ (1:0-0:1). Fraction B (618.5 g) was decolorized on an $\mathrm{MCI}^{\circledR}$ GEL and eluted with $90 \%$ methanol- $\mathrm{H}_{2} \mathrm{O}$ to yield fractions B1-B4. Fractions B1 (116 g) and B2 (135 g) were further separated by repeated silica gel column chromatography to isolate LK-A (20 g). The LK-A powder was dissolved in dimethyl sulfoxide (DMSO) at a concentration of $50 \mathrm{mM}$ and then stored at $-20^{\circ} \mathrm{C}$. Before each experiment, we would freshly dilute the LK-A in medium to achieve the working concentrations in this study. The DMSO concentration was kept below $0.1 \%$ when used in cell culture and did not exert any detectable effect on cell growth or death. Cell culture reagents, including RPMI 1640 medium, Dulbecco's modified Eagle's medium (DMEM)/F12, recombinant human basic fibroblast growth factor (bFGF), recombinant human epidermal growth factor (EGF) and B-27 $7^{\circledR}$ Supplement were purchased from Invitrogen (Thermo Fisher Scientific, Inc., Waltham, MA, USA). The following monoclonal antibodies were used for western blotting: Anti-c-myc (1:1,000; catalog no., 5605S; Cell Signaling Technology, Inc., Danvers, MA, USA), anti-fibronectin (1:2,000; catalog no., 610077; BD Biosciences, Franklin Lakes, NJ, USA), anti- $\beta$-actin $(1: 1,000$; catalog no., 66009-1-1g; Proteintech Group, Chicago, IL, USA) and anti- $\alpha$-tubulin (1:3,000; catalog no., sc-8035; Santa Cruz Biotechnology, Inc., Dallas, TX, USA). All other chemicals, including bovine serum albumin, protease inhibitor cocktail, PBS and Tween-20, were purchased from Sigma-Aldrich (Merck Millipore, Darmstadt, Germany).

Cell culture. S18 and S26 cells are clones of the human NPC cell line CNE2. A stable radioresistant NPC cell line (Sune2-IR) and its parental cell line (Sune2), and the 5-8F NPC cell line, were supplied by and maintained in the State Key Laboratory of Oncology in South China, Collaborative Innovation Center for Cancer Medicine, Sun Yat-sen University Cancer Center (Guangzhou, China) in RPMI 1640 medium supplemented with 5\% fetal bovine serum (FBS) (Invitrogen; Thermo Fisher Scientific, Inc.), penicillin $(100 \mathrm{U} / \mathrm{ml})$ and streptomycin (100 U/ml). The NPC cell lines were incubated at $37^{\circ} \mathrm{C}$ in $5 \%$ $\mathrm{CO}_{2} / 95 \%$ air.

Nasosphere formation assay. S18 and S26 cells were plated in triplicate at a density of 200 cells per well in ultra-low attachment 6-well plates (Corning Incorporated, Corning, NY, USA), and then cultured in DMEM/F12 with $20 \mathrm{ng} / \mathrm{ml} \mathrm{recom}-$ binant human bFGF, $20 \mathrm{ng} / \mathrm{ml}$ recombinant human EGF and
B-27 ${ }^{\circledR}$ Supplement. The spheres were collected after 7 days and counted under a light microscope. Then, we dissociated the primary sphere cells into single-cell suspensions, which were cultured to allow the regeneration of spheres.

SP assay. SP cell analysis and isolation were performed by fluorescence-activated cell sorting (FACS) (Beckman Coulter, Inc., Brea, CA, USA). Before SP cell analysis, cells were pretreated with different concentrations of LK-A for $48 \mathrm{~h}$. Subsequently, the cells were resuspended at a density of $1 \times 10^{6}$ cells $/ \mathrm{ml}$ in RPMI 1640 supplemented with $2 \%$ FBS. Then, cells were incubated with $5 \mu \mathrm{g} / \mathrm{ml}$ Hoechst 33342 (Sigma-Aldrich; Merck Millipore) either alone or with $100 \mu \mathrm{g} / \mathrm{ml}$ verapamil (Sigma-Aldrich; Merck Millipore) at $37^{\circ} \mathrm{C}$ in the dark for $90 \mathrm{~min}$. Cells were washed, centrifuged at $1811 \mathrm{x} \mathrm{g}$ for $10 \mathrm{~min}$ at $4^{\circ} \mathrm{C}$ and resuspended in cold PBS. All cells were kept at $4^{\circ} \mathrm{C}$ in the dark before FACS analysis using dual wavelength analysis.

MTT cell viability assay. First, 2,000 cells were seeded into 96-well plates, incubated overnight and then treated with various concentrations of LK-A for $48 \mathrm{~h}$. Then, $20 \mu \mathrm{l}$ of MTT $(5 \mathrm{mg} / \mathrm{ml})$ was added to each well, and the plate was incubated at $37^{\circ} \mathrm{C}$ for $4 \mathrm{~h}$. Subsequently, the supernatant was carefully removed, and $150 \mu \mathrm{l} /$ well DMSO was added to dissolve the formazan crystals. The absorbance of the soluble product was measured with a microplate spectrophotometer at $490 \mathrm{~nm}$ ( $\mu$ Quant $^{\mathrm{TM}}$; Biotek Instruments, Inc., Winooski, VT, USA). This experiment was performed in six replicates and repeated three times. We calculated the percentage of cell viability for each concentration of LK-A by using the following formula: Cell viability $(\%)=$ A570 nm (sample) $/$ A570 (control DMSO) $x$ 100. The half maximal inhibitory concentration $\left(\mathrm{IC}_{50}\right)$ was determined with GraphPad Prism 5 (GraphPad Software, Inc., La Jolla, CA, USA).

Drug interaction analysis. S18 and S26 cells were counted, plated in triplicate at 2,000 cells per well $(200 \mathrm{ml})$ in 96 -well plates, and allowed to grow overnight. For the experiment group, a concentration gradient of cisplatin (DDP; Jiangsu Hansoh Pharmaceutical Group Co., Ltd., Lianyungang, China) was added to the wells. For the combination groups, $1.0 \mu \mathrm{M}$ LK-A was mixed with a concentration gradient of DDP and then added to the wells. Cell viability was measured $48 \mathrm{~h}$ later by adding an MTT solution. The observation value was detected at $490 \mathrm{~nm}$. The results from the assays were analyzed for the combination effect between LK-A and DDP according to the method described by Jin (17). The following formula was used: $\mathrm{Q}=\mathrm{Ea}+\mathrm{b} /[\mathrm{Ea}+\mathrm{Eb}-(\mathrm{Ea} \times \mathrm{Eb})]$, where $\mathrm{Ea}+\mathrm{b}, \mathrm{Ea}$ and $\mathrm{Eb}$ are the average effects of the combination treatment, LK-A only and DDP only, respectively (17). Q $<0.85$ indicates antagonism, $0.85 \leq \mathrm{Q}<1.15$ indicates additivity and $\mathrm{Q} \geq 1.15$ indicates synergism.

Radiation clonogenic cell survival assay. The NPC cells were seeded into six-well plates with 400 cells per well. Twenty-four hours after plating, LK-A was added at a concentration of $0.4 \mu \mathrm{M}$. After 24 -h exposure to LK-A, the Sune 2 cells and the radioresistant Sune2-IR cells were irradiated at doses of 0,1 , 2 and 3 Gy. Immediately following irradiation, the cells were 
A

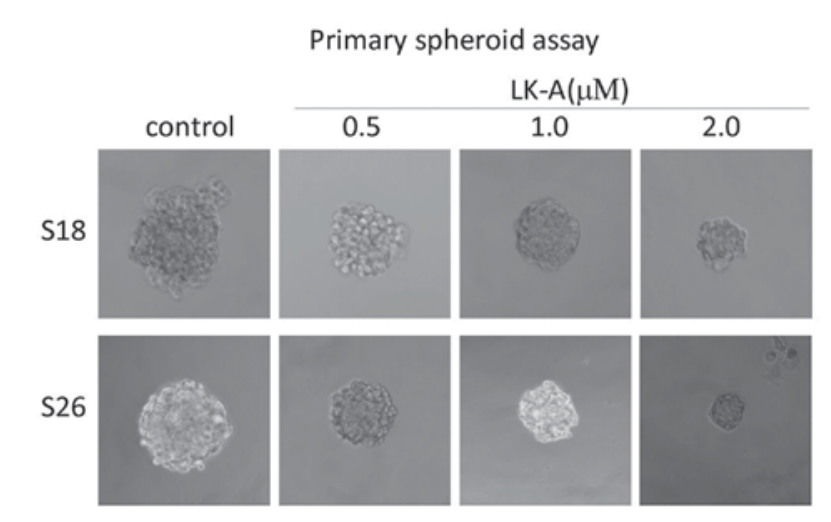

C

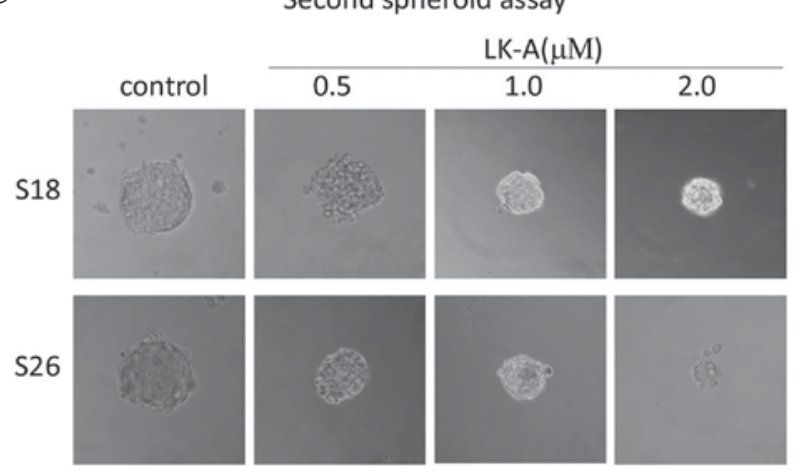

B

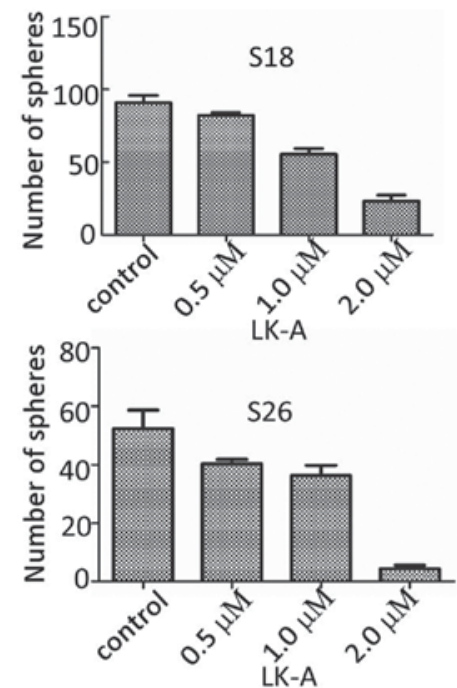

D

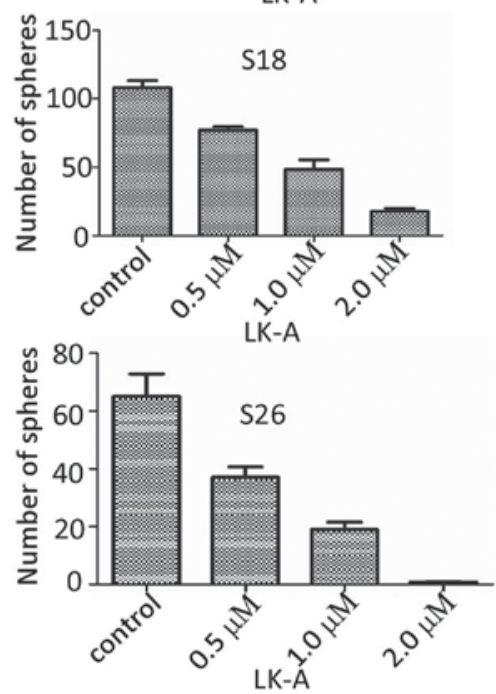

Figure 1. (A and B) LK-A reduced the number and size of the primary spheroids formed. Magnification, x200. S18 and S26 cells were plated in single-cell suspensions at a density of 100 cells/ml in cancer stem cell medium (Dulbecco's modified Eagle's medium/F12, human epidermal growth factor and B27 supplement) and treated with various doses of LK-A for 7 days. (C and D) Primary spheres were harvested, and then re-plated after dilution to analyze the subsphere forming ability. Magnification, x200. LK-A, longikaurin A.

incubated for 7 days at $37^{\circ} \mathrm{C}$ to allow colony formation in a $5 \% \mathrm{CO}_{2}$ environment. After 10 days, the colonies were washed with PBS, fixed with pure ethanol and stained with $0.5 \%$ crystal violet. Colonies containing $>50$ cells were counted as clonogenic survivors. Each point on the survival curves represents the mean surviving fraction (SF) from at least three dishes. The equation $\mathrm{SF}=1-\left(1-\mathrm{e}^{\mathrm{D} / \mathrm{D} 0}\right)^{\mathrm{N}}$ was applied to calculate several parameters, including the cellular radiosensitivity (mean lethal dose, D0), the capacity for sublethal damage repair (quasi-threshold dose, Dq) and the extrapolation number (N). Those parameters were used to calculate the sensitization enhancement ratio (SER) and the SF2 (the SF after irradiation at a dose of 2 Gy).

Western blotting. Cells were seeded into 6-well plates and incubated overnight. Then, the cells were treated with various concentrations of LK-A for $48 \mathrm{~h}$. We used a previously described western blotting method (18). Briefly, equal amounts of protein were separated by $9 \%$ SDS-PAGE and electrophoretically transferred onto polyvinyl difluoridine membranes. Rabbit anti-human c-myc antibody (1:1,000; Cell Signaling
Technology, Inc.) and mouse anti-human fibronectin antibody (1:2,000; BD Biosciences) were used to detect the expression of c-myc and fibronectin, and were incubated with the membranes for $12 \mathrm{~h}$ at $4^{\circ} \mathrm{C}$. Anti-rabbit immunoglobulin (Ig) $\mathrm{G}$ and anti-mouse $\mathrm{IgG}$ secondary antibodies were also used, and $\alpha$-tubulin was used as an internal control. A chemiluminescence substrate and photographic X-ray imaging was used for visualization.

Statistical analysis. Data are presented as the mean \pm standard deviation. GraphPad Prism 5 (GraphPad Software, Inc., La Jolla, CA, USA) was used to determine $\mathrm{IC}_{50}$, and SigmaPlot v10.0 (Systat Software, Inc., San Jose, CA, USA) was used to construct cell survival curves. Student's t-test was used to compare the means of various groups. $\mathrm{P}<0.05$ was considered to indicate a statistically significant difference.

\section{Results}

LK-A inhibits the self-renewal capacity of NPC S18 and S26 cells. To investigate the effect of LK-A on the self-renewal 
Table I. $\mathrm{IC}_{50}$ value for DDP in the presence or absence of LK-A in S26, S18 and 5-8F cells.

\begin{tabular}{lcrr}
\hline $\mathrm{IC}_{50}$ value for DDP in the cell lines $(48 \mathrm{~h})$ & $\mathrm{S} 18$ & $\mathrm{~S} 26$ & $5-8 \mathrm{~F}$ \\
\hline Control & $10.10 \pm 1.00 \mu \mathrm{M}$ & $11.45 \pm 1.06 \mu \mathrm{M}$ & $8.00 \pm 0.90 \mu \mathrm{M}$ \\
$1 \mu \mathrm{M}$ LK-A & $7.00 \pm 0.85 \mu \mathrm{M}$ & $7.12 \pm 0.85 \mu \mathrm{M}$ & $2.65 \pm 0.42 \mu \mathrm{M}$
\end{tabular}

$\mathrm{IC}_{50}$, half maximal inhibitory concentration; LK-A, longikaurin A; DDP, cisplatin.

A

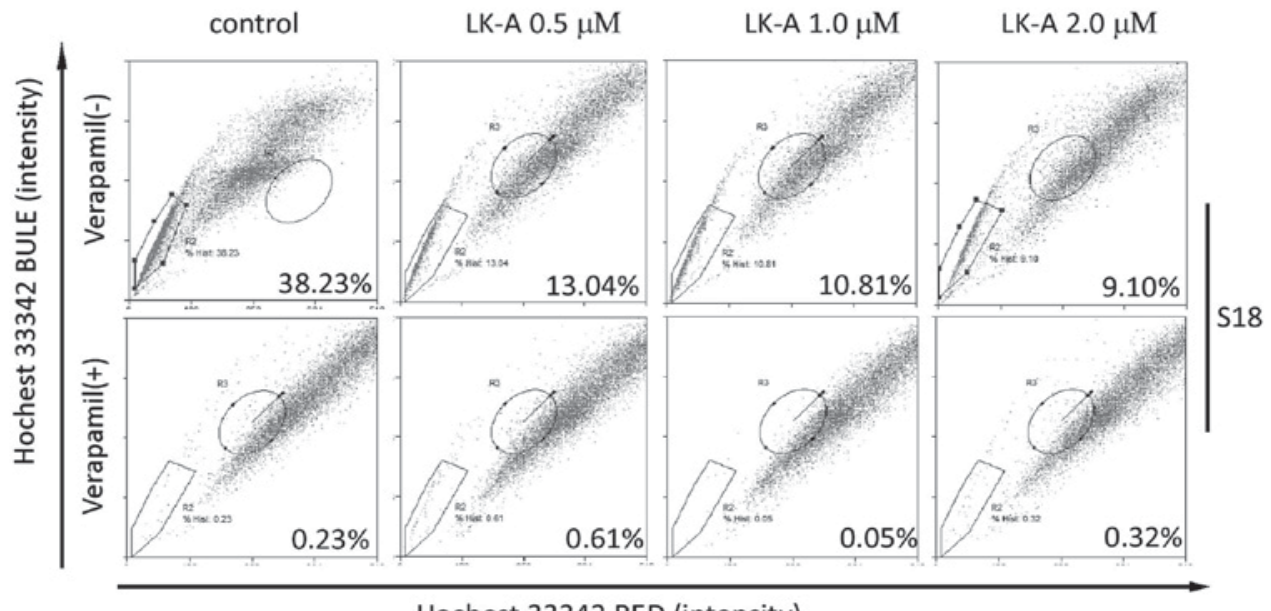

Hochest 33342 RED (intensity)

B

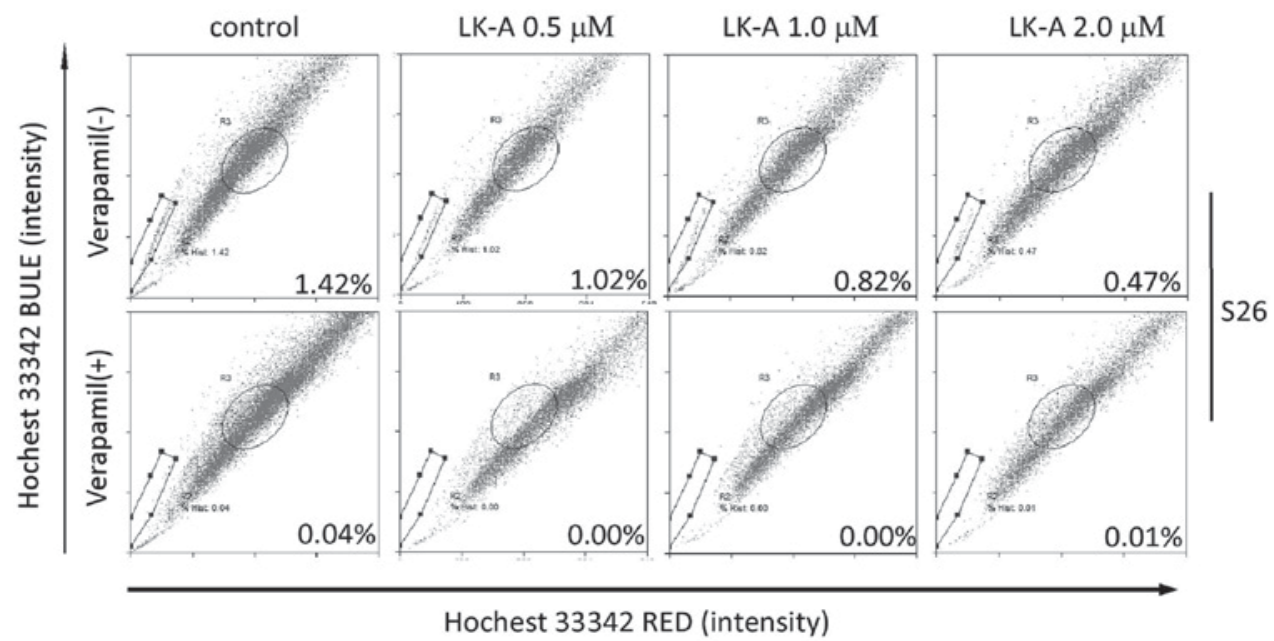

Figure 2. LK-A decreases the percentage of SP cells in the S18 cell line. (A and B) Percentages of SP cells in the (A) S18 and (B) S26 cell lines after treatment with LK-A. The SP cell profiles in the presence of verapamil are shown in the bottom panels. The percentages of SP cells are indicated. LK-A, longikaurin A; SP, side population.

ability of NPC CSCs, we evaluated the sphere-forming capacity after adding LK-A to S18 and S26 cells growing in serum-free non-adherent medium. The result revealed that interference by LK-A resulted in markedly diminished sphere size and number of spheres in a dose-dependent manner in both S18 and S26 cells (Fig. 1A and B).

To further investigate the effect of LK-A on second-generation spheres, after spheres formation, we dissociated the sphere cells into single cells, and we cultivated these single cells in serum-free non-adherent culture with various concentrations of LK-A again. The first-generation spheres were capable of generating second-generation spheres, suggesting that NPC sphere-generated cells have the capacity of self-renewal (Fig. 1C). Meanwhile, we observed that second-generation spheres formed more spheres than primary spheres (Fig. 1B and D). These second-generation spheres were observed to have decreased sphere-forming efficiency under treatment with increasing concentrations of LK-A, with smaller sphere size and lower number of spheres (Fig. 1C and D). These data indicated that LK-A inhibited the self-renewal capacity of NPC cells in a dose-dependent manner.

$L K$-A decreases the percentage of SP cells in the S18 cell line. SP cells are one subpopulation of the cancer cells that effluxes 
A

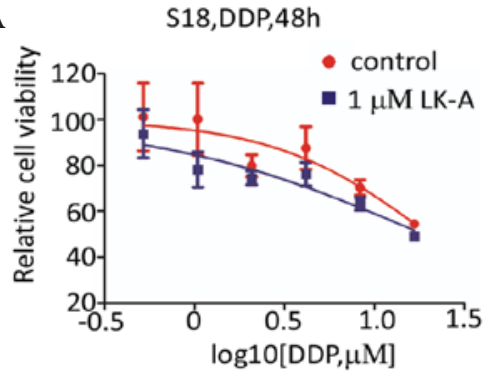

B

D

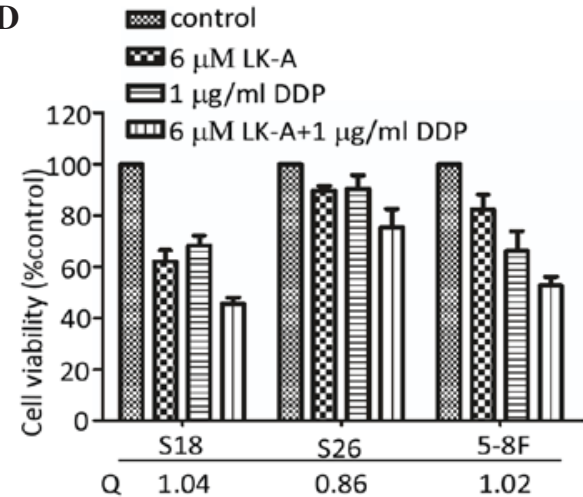

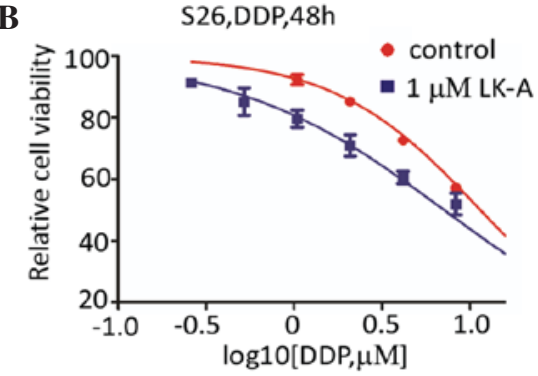

$\mathbf{E}$

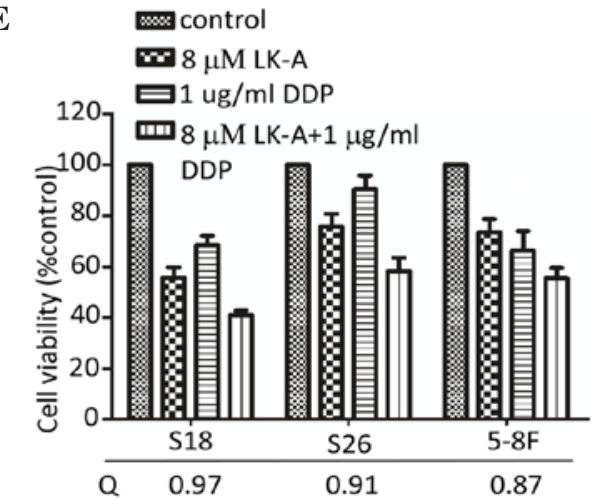

Figure 3. Cytotoxic effect of cisplatin alone or in combination with LK-A. (A-E) Shifting of dose-response curves of cisplatin by LK-A. Three nasopharyngeal carcinoma cell lines, (A) S18, (B) S26 and (C) 5-8F, were assayed by MTT assay. (D) LK-A potentiated the effect of cisplatin in the three cell lines. $\mathrm{Q}=\mathrm{Ea}+\mathrm{b} /(\mathrm{Ea}+\mathrm{Eb}-\mathrm{EaxEb})$, where $\mathrm{Ea}+\mathrm{b}, \mathrm{Ea}$ and $\mathrm{Eb}$ are the average effects of the combination treatment, LK-A only and cisplatin only, respectively. Q $<0.85$ indicates antagonism, $0.85 \leq \mathrm{Q}<1.15$ indicates additivity and $\mathrm{Q} \geq 1.15$ indicates synergism. LK-A, longikaurin A; DDP, cisplatin.

the DNA binding dye Hoechst 33342 out of the cell membrane. Previous studies have shown that the SP cells have stem cell characteristics and enrich the stem cell population in NPC (19). To investigate whether LK-A could impact the percentage of the SP cells in NPC cells, we measured the SP cell percentage of S18 and S26 cells treated with LK-A for $48 \mathrm{~h}$. S18 and S26 are two cell lines originated from CNE2, and S18 has a higher stemness characteristic with a high SP cell proportion, while S26 has a lower stemness characteristic with a lower SP cell proportion (13). As shown in Fig. 2A and B, both in S18 and S26 cells, SP cells are blocked by verapamil at a final concentration of $100 \mu \mathrm{g} / \mathrm{ml}$. These results indicated that the percentage of SP cells was $\sim 26$-fold higher in S18 cells than in S26 cells (38.23 vs. $1.47 \%$ ). Upon treatment with LK-A, the percentages of SP cells in S18 and S26 cells were all decreased (Fig. 2A and B). S18 cells showed a 2.93-fold decrease in the SP cell proportion upon addition of $0.5 \mu \mathrm{M}$ LK-A (Fig. 2A). Taken together, these results indicate that LK-A could decrease the percentage of the SP cells in NPC cells.

LK-A potentiates the additivity effect of DDP in NPC cells. $\mathrm{CSCs}$ are believed to be the cause of chemotherapy resistance (6). Chemotherapy is not effective for a large number of patients with NPC. As LK-A could decrease the proportion of SP cells in NPC cells, we attempted to determine whether LK-A could increase the chemotherapy sensitivity to DDP of NPC cells. As shown in Fig. 3A-C, the dose-response curves of DDP with $1 \mu \mathrm{M}$ LK-A were obviously shifted to the left in NPC cells. The $\mathrm{IC}_{50}$ value for DDP in the presence of LK-A in S26, S18 and 5-8F cells was significantly reduced (Table I). According to the method by Jin (17), the Q value was 1.04 in S18 cells, 0.86 in S26 cells and 1.02 in 5-8F cells at the concentration of $6.0 \mu \mathrm{M}$ LK-A (Fig. 3D). As shown in Fig. 3E, at a higher concentration of LK-A $(8.0 \mu \mathrm{M})$, the Q value was 0.97 in S18 cells, 0.91 in S26 cells and 0.87 in 5-8F cells. To the best of our knowledge, $\mathrm{Q}<0.85$ indicates that the combination of two drugs has an effect of antagonism, while a $Q$ value between 0.85 and 1.15 indicates an additivity effect and $Q>1.15$ means synergism. Therefore, these data suggested that LK-A has an additive effect to that of DDP in the treatment of NPC cells.

$L K$-A sensitizes NPC cells to radiation and reverses radioresistance in NPC cells. Despite improvements in radiation technology, local recurrence or metastasis occurs in a high proportion of NPC patients due to radioresistance (20). According to the experiment results described above, we aimed to investigate whether LK-A could sensitize the radioresistance of NPC cells. The Sune2-IR radioresistant cell line was obtained from Sune 2 cells exposed to ionizing radiation (cumulative dose is $28 \mathrm{~Gy}$ ). Using the clonogenic survival assay, we found a marked reduction in clone formation in Sune2 and Sune2-IR cells after irradiation combined with the intervention of LK-A (Fig. 4A). Each point on the survival curve represents the mean $\mathrm{SF}$ from triplicate experiments (Fig. 4B). There was a significant difference in SF between parental and radioresistant cells at 1,2 and 3 Gy of radiation, indicating that the Sune2-IR cells were more radiation resistant than the Sune2 cells. Additionally, LK-A $(0.4 \mu \mathrm{M})$ shifted the Sune2 and Sune2-IR cell dose survival curves clearly to the left, which indicated the increased radiosensitivity of Sune2 and Sune2-IR cells caused by LK-A treatment (Fig. 4B). Sune2 and Sune2-IR cells treated with LK-A $(0.4 \mu \mathrm{M})$ had a SER of 1.16 and 1.14, respectively (Fig. 4C). The survival curve 
Table II. Clonogenic survival parameters of Sune2 and Sune2-IR cells after LK-A exposure.

\begin{tabular}{lcccccc}
\hline Cell lines & Groups & D0 & Dq & SF2 & N & SER (Dq) \\
\hline Sune2 & Control & 2.58 & 1.17 & 0.59 & 1.25 & 1.16 \\
& $0.4 \mu$ M LK-A & 2.48 & 1.01 & 0.53 & 0.63 & 2.04 \\
Sune2-IR & Control & 2.22 & 1.51 & 0.58 & 1.76 \\
& $0.4 \mu$ M LK-A & 2.15 & 1.33 & & \\
\hline
\end{tabular}

LK-A, longikaurin A; D0, mean lethal dose; Dq, quasi-threshold dose; SF, surviving fraction; SF2, SF after irradiation at a dose of 2 Gy; N, extrapolation number; SER, sensitization enhancement ratio.

A
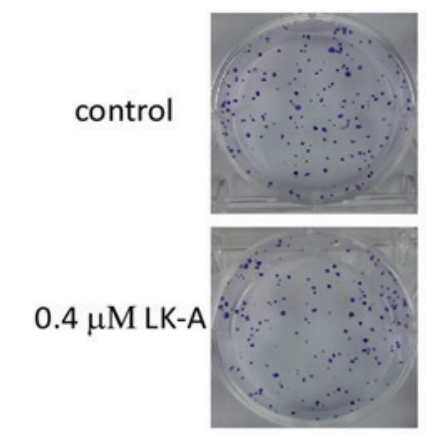

control

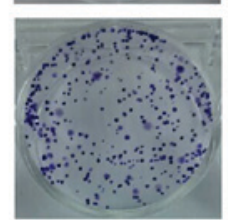

$0.4 \mu \mathrm{M}$ LK-A

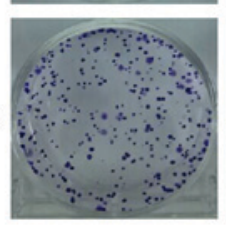

B

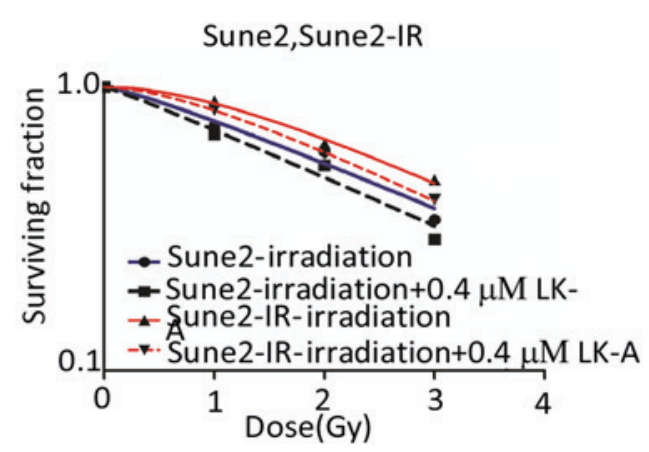

$1 \mathrm{~Gy}$
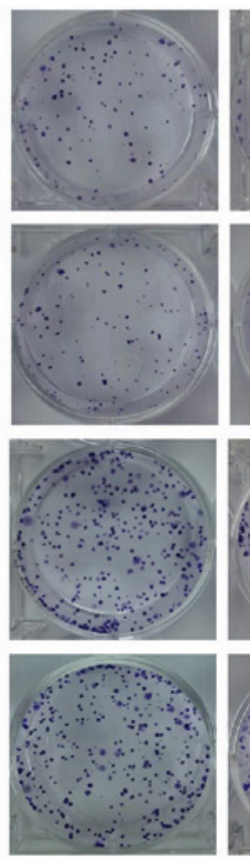

C

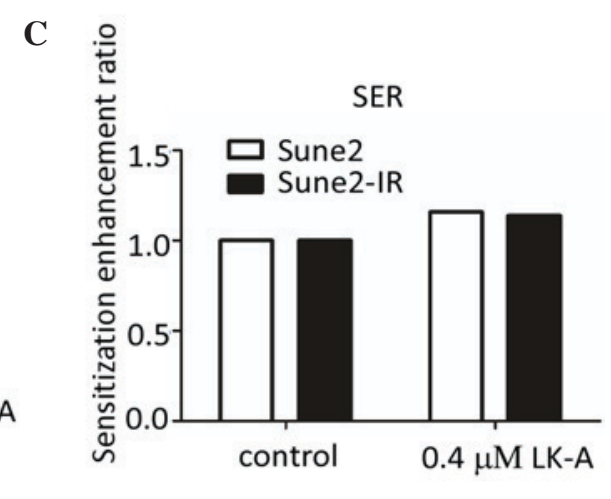

Figure 4. LK-A pretreatment sensitized Sune2 cells to radiation and reversed the radioresistance of Sune2-IR cells. (A) LK-A was added in concentrations of $0.4 \mu \mathrm{mol} / 1$. After $24 \mathrm{~h}$ exposure to LK-A, the Sune 2 cells and the radioresistant Sune2-IR cells were irradiated at doses of 0, 1,2 and 3 Gy. After 10 days, the colonies were fixed with pure ethanol and stained with $0.5 \%$ crystal violet. (B) Clonogenic survival assays of Sune 2 and Sune2-IR cells pretreated with LK-A. Surviving fractions were calculated by the number of colonies divided by the number of seeded cells multiplied by the plating efficiency. (C) SER was calculated from the data shown in A. LK-A, longikaurin A; SER, sensitization enhancement ratio.

parameters are listed in Table II. All parameters of the LK-A groups were smaller than those of the control groups.

It was reported that silencing fibronectin extra domain $\mathrm{A}$ (EDA) enhances radiosensitivity in NPC (21). Therefore, we performed western blotting to detect the protein expression of fibronectin in the Sune2 and Sune2-IR cell lines. Fig. 5A demonstrates that Sune2-IR cells expressed fibronectin at a higher level than Sune2 cells. These results demonstrated that LK-A could sensitize the radioresistance of NPC cells to irradiation. 


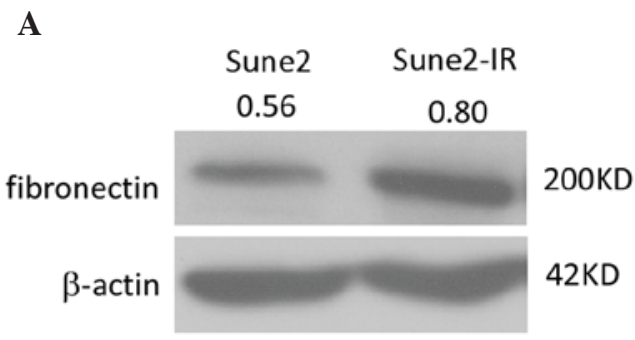

B S18 S26

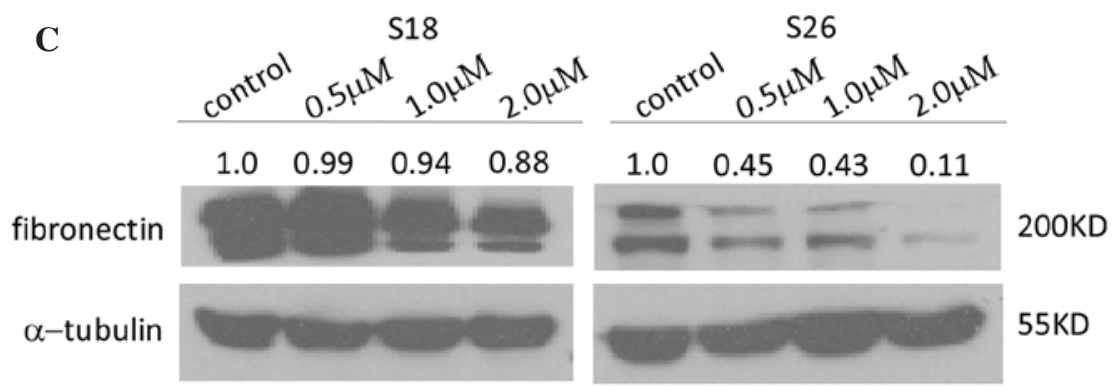

Figure 5. Longikaurin A pretreatment downregulated the protein expression of the (A) c-myc and (B) fibronectin in the S18 and S26 cell lines. (C) Expression of fibronectin in the Sune2 and Sune2-IR cell lines. Numbers in the figure refer to mean grey value.

LK-A downregulates c-myc and fibronectin. As it is well known that there are not reliable CSC markers in NPC cells (22), we investigated whether LK-A could influence the expression of stem cell markers in NPC cells. The expression of Nanog, sex determining region Y-box 2 and octamer-binding transcription factor 4 could not be detected in the S18 or S26 cell lines. In addition, the expression of the Bmi-1, ATP-binding cassette (ABC) $\mathrm{G} 2$ and $\beta$-catenin were not downregulated by the interference of LK-A (data not show). However, we could detect that LK-A decreased the expression of c-myc in NPC cells (Fig. 5B). c-myc is sufficient to impart high self-renewal and tumor-propagating capacities in breast cancer (23), and overexpression of c-myc increases the stemness of NPC cells (24). These data indicated that LK-A may influence the stemness of NPC cells by downregulating the expression of c-myc.

As aforementioned, LK-A may sensitize Sune2 cells to radiation and reversed the radioresistance of Sune2-IR cells through influencing fibronectin protein expression. Fibronectin is known to play important roles in angiogenesis, lymphangiogenesis and metastasis in malignant tumors (25). Therefore, we wondered whether LK-A treatment could downregulate the protein expression of fibronectin in NPC cells. As shown in Fig. 5C, LK-A treatment downregulated the protein expression of fibronectin in a dose-dependent manner. These data indicated that LK-A may impair radioresistance by downregulation of fibronectin.

\section{Discussion}

Currently, the main treatments of NPC are radiotherapy and platinum-based chemotherapy (3). However, patients still relapse from primary treatment with radiotherapy or chemo-irradiation, and some NPC patients that are diagnosed at advanced stages fail to react to the treatment and succumb to cancer progression (26). Therefore, CSCs, which should be responsible for the malignant neoplasm recurrence and metastasis (5), may be a novel therapy target for NPC. In addition, natural products and their derivatives act as new therapy drugs to target the NPC CSCs.

As a natural ent-kaurene diterpenoid, LK-A has been reported to induce apoptosis in multiple myeloma $\mathrm{H} 929$ cells, hepatocellular carcinoma cells and NPC cells $(15,16,27)$. However, there are limited studies on the role of LK-A in targeting CSCs. Our recent study revealed that LK-A inhibited the colony formation ability of NPC cells at a low concentration and exhibited anti-tumor activity in CNE2 xenograft tumor models (15). This may be due to a key role of LK-A in targeting the NPC CSCs. In the present study, in a dose-dependent manner, LK-A reduced the nasosphere formation rate, and decreased the number and volume of the primary spheres. Moreover, the secondary nasosphere formation efficiency of S18 and S26 cells was also impaired following treatment with LK-A (Fig. 1A-D). To the best of our knowledge, SP cells have numerous stem cell properties, including unlimited proliferation potential, self-renewal, differentiation, resistance to chemotherapy and radiation, and a strong tumor formation ability in vivo (19). According to Fig. 2A, LK-A could decrease the percentage of the SP cells in the S18 cells. Due to the low numbers of SP cells in S26 cells, we found that LK-A could not increase the percentage of SP cells (Fig. 2B). Together, these data suggest that LK-A suppressed the stemness of NPC cells in vitro.

In addition to serving as a potential therapeutic for NPC CSCs, our results suggest that LK-A exerts additive effects with DDP on NPC cells (Fig. 3A-E). Radiotherapy is known as the main standard treatment of NPC. Therefore, we wonder whether LK-A could enhance the efficacy of radiation in NPC cells. The results demonstrated that LK-A treatment not only could sensitize Sune2 cells to radiation, but also reverse the radioresistance of Sune2-IR cells (Fig. 4A-C).

Previous studies indicated that silencing fibronectin EDA increases radiosensitivity in NPC involving the focal adhesion 
kinase/Akt/c-Jun N-terminal kinase pathway (20). According to Fig. 5A, LK-A may enhance the radiation efficacy though downregulation of fibronectin. Moreover, to the best of our knowledge, fibronectin includes three components: EDA, EDB and connecting segment III, an important extracellular matrix glycoprotein in the tumor microenvironment (28). It has been recently reported that fibronectin can enhance the formation of multi-cellular spheroids in ovarian cancer (29). Therefore, as expected, the protein expression level of fibronectin decreased, which was induced by LK-A, in NPC cells (Fig. 3B). However, the mechanism requires to be further investigated.

Previous research about LK-A indicated that LK-A induces $\mathrm{G} 2 / \mathrm{M}$ phase arrest via downregulation of S-phase kinase-associated protein 2 (Skp2) and apoptosis induction in hepatocellular carcinoma cells (17). Recent studies suggest that Skp2 regulates the NPC CSC maintenance (30). However, Skp2 was not affected in NPC cells treated with LK-A.

Furthermore, we explored the effects of LK-A on stem cell markers. Despite the fact that LK-A treatment decreased the percentage of SP in NPC cells, the expression of ABCG2 protein was not affected (data not show). However, we found that LK-A reduced the expression of c-myc in a dose-dependent manner (Fig. 5B and C). Meanwhile, c-myc is one member of the myc oncoprotein family, whose role in the pathogenesis of numerous human neoplastic diseases has received wide empirical support. It is largely believed that c-myc plays an important role in carcinogenesis and tumor progression due to its influence on all basic cellular processes (31). In our study, both S18 and S26 cells overexpressed c-myc. Following treatment with LK-A, the expression of c-myc was clearly downregulated (Fig. 5B). The self-renewal ability of cells can be verified by spheroid formation assay. Previous studies have indicated that c-myc serves a key role in cooperative actions with p53 and phosphatase and tensin homolog in the regulation of normal and malignant stem/progenitor cell differentiation, self-renewal and tumorigenic potential (32). Therefore, LK-A may affect the self-renewal ability of NPC cells through c-myc. Moreover, c-myc could promote radioresistance in $\mathrm{PKH} 26+$ NPC cells, which are enriched for a stem cell-like subpopulation and are resistant to radiotherapy (24). Therefore, LK-A enhances radiosensitivity in NPC due to fibronectin and c-myc. However, the mechanism by which LK-A influences the expression of c-myc requires to be further investigated.

In summary, LK-A could target CSCs in NPC cells, and it could enhance the efficacy of chemotherapy drugs and radiotherapy in vitro. However, future studies are required to clarify the mechanisms by which LK-A targets CSCs. Determination of the feasibility of the clinical application of this compound is also important for the treatment of NPC patients.

\section{References}

1. Chang ET and Adami HO. The enigmatic epidemiology of nasopharyngeal carcinoma. Cancer Epidemiol Biomarkers Prev 15: 1765-1777, 2006.

2. Wei KR, Zheng RS, Zhang SW, Liang ZH, Ou ZX and Chen WQ: Nasopharyngeal carcinoma incidence and mortality in China in 2010. Chin J Cancer 33: 381-387, 2014.

3. Xu T, Tang J, Gu M, Liu L, Wei W and Yang H: Recurrent nasopharyngeal carcinoma: A clinical dilemma and challenge. Curr Oncol 20: e406-e419.
4. Wang C (ed): Radiation Therapy for Head and Neck Neoplasms. 3rd edition. Wiley-Liss, New York, NY, 1997.

5. Zhang Z, Filho MS and Nör JE: The biology of head and neck cancer stem cells. Oral Oncol 48: 1-9, 2012.

6. Clarke MF, Dick JE, Dirks PB, Eaves CJ, Jamieson $\mathrm{CH}$, Jones DL, Visvader J, Weissman IL and Wahl GM: Cancer stem cells-perspectives on current status and future directions: AACR Workshop on cancer stem cells. Cancer Res 66: 9339-9344, 2006.

7. Ke J, Wu X, Wu X, He X, Lian L, Zou Y, He X, Wang H, Luo Y, Wang L and Lan P: A subpopulation of CD24+ cells in colon cancer cell lines possess stem cell characteristics. Neoplasma 59: 282-288, 2012.

8. Jaggupilli A and Elkord E: Significance of CD44 and CD24 as cancer stem cell markers: An enduring ambiguity. Clin Dev Immunol 2012: 708036, 2012.

9. Ricardo S, Vieira AF, Gerhard R, Leitão D, Pinto R, Cameselle-Teijeiro JF, Milanezi F, Schmitt F and Paredes J: Breast cancer stem cell markers CD44, CD24 and ALDH1: Expression distribution within intrinsic molecular subtype. J Clin Pathol 64: 937-946, 2011.

10. Wang Q, Chen ZG, Du CZ, Wang HW, Yan L and Gu J: Cancer stem cell marker CD133+ tumour cells and clinical outcome in rectal cancer. Histopathology 55: 284-293, 2009.

11. Kondo T, Setoguchi T and Taga T: Persistence of a small subpopulation of cancer stem-like cells in the C6 glioma cell line. Proc Natl Acad Sci USA 101: 781-786, 2004.

12. Haraguchi N, Utsunomiya T, Inoue H, Tanaka F, Mimori K, Barnard GF and Mori M: Characterization of a side population of cancer cells from human gastrointestinal system. Stem Cells 24: 506-513, 2006.

13. Deng CC, Liang Y, Wu MS, Feng FT, Hu WR, Chen LZ, Feng QS, Bei JX and Zeng YX: Nigericin selectively targets cancer stem cells in nasopharyngeal carcinoma. Int J Biochem Cell Biol 45: 1997-2006, 2013.

14. Wu MS, Wang GF, Zhao ZQ, Liang Y, Wang HB, Wu MY, Min P, Chen LZ, Feng QS, Bei JX, et al: Smac mimetics in combination with TRAIL selectively target cancer stem cells in nasopharyngeal carcinoma. Mol Cancer Ther 12: 1728-1737, 2013.

15. Zou QF, Du JK, Zhang H, Wang HB, Hu ZD, Chen SP, Du Y, Li MZ, Xie D, Zou J, et al: Anti-tumour activity of longikaurin A (LK-A), a novel natural diterpenoid, in nasopharyngeal carcinoma. J Transl Med 11: 200, 2013.

16. Liao YJ, Bai HY, Li ZH, Zou J, Chen JW, Zheng F, Zhang JX, Mai SJ, Zeng MS, Sun HD, et al: Longikaurin A, a natural ent-kaurane, induces $\mathrm{G} 2 / \mathrm{M}$ phase arrest via downregulation of Skp2 and apoptosis induction through ROS/JNK/c-Jun pathway in hepatocellular carcinoma cells. Cell Death Dis 5: e1137, 2014.

17. Jin ZJ: About the evaluation of drug combination. Acta Pharmacol Sin 25: 146-147, 2004

18. Cao JY, Liu L, Chen SP, Zhang X, Mi YJ, Liu ZG, Li MZ, Zhang H, Qian CN, Shao JY, et al: Prognostic significance and therapeutic implications of centromere protein $\mathrm{F}$ expression in human nasopharyngeal carcinoma. Mol Cancer 9: 237, 2010.

19. Wang J, Guo LP, Chen LZ, Zeng YX and Lu SH: Identification of cancer stem cell-like side population cells in human nasopharyngeal carcinoma cell line. Cancer Res 67: 3716-3724, 2007.

20. Guo Y, Zhu XD, Qu S, Li L, Su F, Li Y, Huang ST and Li DR: Identification of genes involved in radioresistance of nasopharyngeal carcinoma by integrating gene ontology and protein-protein interaction networks. Int J Oncol 40: 85-92, 2012.

21. Ou J, Pan F, Geng P, Wei X, Xie G, Deng J, Pang X and Liang H: Silencing fibronectin extra domain A enhances radiosensitivity in nasopharyngeal carcinomas involving an FAK/Akt/JNK pathway. Int J Radiat Oncol Biol Phys 82: e685-e691, 2012.

22. Wei P, Niu M, Pan S, Zhou Y, Shuai C, Wang J, Peng S and Li G. Cancer stem-like cell: A novel target for nasopharyngeal carcinoma therapy. Stem Cell Res Ther 5: 1-9, 2014.

23. Nair R, Roden DL, Teo WS, McFarland A, Junankar S, Ye S, Nguyen A, Yang J, Nikolic I, Hui M, et al: c-Myc and Her2 cooperate to drive a stem-like phenotype with poor prognosis in breast cancer. Oncogene 33: 3992-4002, 2014.

24. Wang WJ, Wu SP, Liu JB, Shi YS, Huang X, Zhang QB and Yao KT: MYC regulation of $\mathrm{CHK} 1$ and $\mathrm{CHK} 2$ promotes radioresistance in a stem cell-like population of nasopharyngeal carcinoma cells. Cancer Res 73: 1219-1231, 2013.

25. Ou JJ, Wu F and Liang HJ: Colorectal tumor derived fibronectin alternatively spliced EDA domain exserts lymphangiogenic effect on human lymphatic endothelial cells. Cancer Biol Ther 9: 186-191, 2010. 
26. Xu T, Tang J, Gu M, Liu L, Wei W and Yang H: Recurrent nasopharyngeal carcinoma: A clinical dilemma and challenge. Curr Oncol 20: e406-e419, 2013.

27. Zhao S, Pu JX, Sun HD and Wu YL: Longikaurin A induces apoptosis of multiple myeloma H929 cells. Zhongguo Shi Yan Xue Ye Xue Za Zhi 20: 611-615, 2012 (In Chinese).

28. French-Constant C: Alternative splicing of fibronectin-many different proteins but few different functions. Exp Cell Res 221: 261-271, 1995.

29. Xie XY, Liu SL, Wang H, Lin L, Zhu HM, Zhang JJ and Zheng Y: The influence of fibronectin on the formation of multi-cellular spheroid of ovarian cancer. Sichuan Da Xue Xue Bao Yi Xue Ban 45: 240-244, 2014 (In Chinese).
30. Wang J, Huang Y, Guan Z, Zhang JL, Su HK, Zhang W, Yue CF, Yan M, Guan S and Liu QQ: E3-ligase Skp2 predicts poor prognosis and maintains cancer stem cell pool in nasopharyngeal carcinoma. Oncotarget 5: 5591-5601, 2014.

31. O'Donnell KA, Wentzel EA, Zeller KI, Dang CV and Mendell JT: c-Myc-regulated microRNAs modulate E2F1 expression. Nature 435: 839-843, 2005.

32. Zheng H, Ying H, Yan H, Kimmelman AC, Hiller DJ, Chen AJ, Perry SR, Tonon G, Chu GC, Ding Z, et al: Pten and p53 converge on c-Myc to control differentiation, self-renewal and transformation of normal and neoplastic stem cells in glioblastoma. Cold Spring Harb Symp Quant Biol 73: 427-437, 2008. 